\title{
QUIEBRES EPISTEMOLÓGICOS PARA LA ENSEÑANZA DE LA TEORÍA CUÁNTICA ${ }^{1}$
}

\author{
Zulma Estela Muñoz Burbano \\ Universidad de Nariño
}

\section{RESUMEN}

Este artículo es resultado de la investigación desarrollada dentro del proyecto macro titulado: Enseñanza de la Estructura Atómica de la Materia. La metodología seguida es una adaptación de las metodologías utilizadas en la realización de estados del arte, que se realiza en dos fases: una heurística, o de recopilación de la información, y una fase hermenéutica, que corresponde al análisis de la información y consolidación de la reflexión. El problema planteado es una enseñanza de la Estructura atómica de la materia simplista y de poca profundidad, que no toma como base la Teoría Cuántica.

El documento establece un breve recorrido por la historia de la filosofía y la historia de las ciencias, desde la Grecia Antigua hasta inicios del siglo XX. Parte de la epistemología de Tomas Kuhn, la dinámica de la "ciencia normal" y la disrupción que generan las "revoluciones científicas" y cómo deberían tomarse en cuenta en la enseñanza de la Teoría Cuántica. Dentro de los quiebres epistemológicos que se describen están: el atomismo de Demócrito, el paso del modelo geocéntrico al modelo heliocéntrico y la ruptura en el pensamiento que significa el advenimiento de la Teoría Cuántica, con énfasis en la posibilidad de asumir estas rupturas para la enseñanza de esta teoría. Concluye con la necesidad de una enseñanza que asumiera la historia de las ciencias y la filosofía, con un acento en los quiebres epistemológicos que el paso de un paradigma a otro requiere, especialmente en el quiebre que demanda la Teoría Cuántica y el indeterminismo que le subyace.

Palabras clave: enseñanza, estructura atómica, quiebre epistemológico, teoría cuántica.

1 El artículo es resultado del proyecto de investigación doctoral Enseñanza de la Estructura Atómica de la Materia del programa de Doctorado en en Ciencias de la Educación RUDECOLOMBIA.

2 Estudiante del Doctorado en Ciencias de la Educación RUDECOLOMBIA-Universidad de Nariño. Línea de investigación Enseñanza de las ciencias. Correo electrónico: zulmamu0706@hotmail. com. Código ORCID: https://orcid.org/0000-0003-2325-9405 


\title{
EPISTEMOLIGICAL BREAKS FOR THE TEACHING OF QUANTUM THEORY
}

\begin{abstract}
This article is the result of the research developed within the macro project titled "Teaching of the Atomic Structure of the Matter". The applied methodology is an adaptation of the methodologies utilized in the achievement of states of the art, which includes two steps: a heuristic, or information gathering, and a hermeneutical stage that involves analysis of the information and consolidation of the reflection. The problem addressed in this work is teaching of Atomic structure of the simplistic and shallow matter, without being based on the Quantum Theory.

This document presents a brief overview of the history of Philosophy and Sciences, from ancient Greece to the beginning of the twentieth century. Also, it is discussed a part of the Tomas Kuhn's epistemology, the dynamics of the "normal science" and the disruptions generated by "scientific revolutions", and how these aspects should be articulated in Quantum Theory teaching. Among the epistemological breaks here described are: the atomism of Democritus, the transition from the geocentric model to the heliocentric model and the thought rupture caused by the advent of the Quantum Theory, emphasizing on the possibility of applying these ruptures in the teaching of this theory. The conclusion states the necessity of a teaching method that would take on the history of sciences and philosophy, highlighting the epistemological breaks required for the transition from one paradigm to another, especially on the break that the Quantum Theory requires and the indeterminism that underlies it.
\end{abstract}

Key words: teaching, atomic structure, epistemological break, quantum theory. 


\title{
ROMPIMENTOS EPISTEMOLÓGICOS PARA O ENSINO DA TEORIA QUÂNTICA
}

\begin{abstract}
RESUMO
Este artigo é o resultado de pesquisa desenvolvida dentro do macro projeto intitulado: Ensino da Estrutura Atômica da Matéria. A metodologia seguida é uma adaptação dos métodos utilizados na realização estado da arte, que é realizado em duas fases: uma heurística, ou recolha de informação, e uma fase hermenêutica, o que corresponde a análise da informação e consolidação a reflexão. 0 problema colocado é um ensinamento da estrutura atômica da matéria superficial e simplista, que não toma como base a Teoria Quântica.

O documento fornece uma breve visão geral da história da filosofia e da história da ciência, desde a Grécia antiga até o início do século XX. Parte da epistemologia de Thomas Kuhn, a dinâmica da "ciência normal" e interrupções geradas pelas "revoluções científicas" e como eles devem ser tidos em conta no ensino de teoria quântica. Dentro dos intervalos epistemológicas que são descritos são eles: o atomismo de Demócrito, a passagem do geocêntrica de modelo heliocêntrico e o rompimento no pensamento que significa o advento da teoria quântica, com destaque para a possibilidade de tomar estas pausas para o ensino desta teoria. Conclui-se com a necessidade de educação que assuma a história das ciências e da filosofia, com ênfase nos rompimentos epistemológicos que a passagem de um paradigma para outro exige, especialmente no intervalo exigido pela teoria quântica e indeterminismo atrás dele.
\end{abstract}

Palavras-chave: ensino, estrutura atômica, rompimento epistemológico, teoria quântica. 


\section{INTRODUCCIÓN}

En este artículo de reflexión, se da cuenta de algunos referentes teóricos en torno al proceso de construcción de la ciencia, su relación dialógica con la filosofía y cómo podrían articularse estas construcciones en la enseñanza de la Teoría Cuántica. Es fruto de la investigación documental, adelantada en el proyecto macro titulado: Enseñanza de la Estructura Atómica de la Materia.

Se toma como punto de partida la dinámica de construcción de la ciencia desde la perspectiva de Thomas Kuhn y el concepto de quiebre epistemológico. Pese a que la noción de quiebre epistemológico se ha vinculado a Gaston Bachelard, quien, en La formación del espíritu científico, ${ }^{3}$ establece como necesaria una ruptura entre el pensamiento cotidiano y el conocimiento científico, esta reflexión se acerca más a la posición de Tomas Kuhn, al trazar, en La estructura de las revoluciones científicas, ${ }^{4}$ rupturas, que llevan a que el desarrollo de las ciencias no siguiera un proceso uniforme y lineal basado en un hipotético método científico, sino se trata de un complejo sistema de revoluciones y de estados de ciencia normal y ciencia revolucionaria. Para el epistemólogo:

“Ciencia normal' significa investigación basada firmemente en una o más realizaciones científicas pasadas, realizaciones que alguna comunidad científica particular reconoce, durante cierto tiempo, como fundamento para su práctica posterior". 5

La ciencia normal es una ampliación de conocimiento, pues los supuestos básicos del paradigma no se constituyen como problemáticos y se trabaja bajo un mismo marco teórico y metodológico. Sin embargo, según Kuhn, tarde o temprano comenzarán a presentar anomalías que se resisten a que se resolvieran bajo el paradigma establecido. Comienza un periodo de crisis, donde el valor epistémico del paradigma vigente se examinará; en esta etapa, puede necesitarse la construcción de estructuras teóricas alternativas, que no siempre corresponden a una reorganización, sino se trata de una ruptura, que constituye el quiebre epistemológico, por afectar las bases teóricas del paradigma en crisis.

La concepción de la construcción de ciencia como un proceso dinámico, en el que una teoría o un campo de investigación, para que se aceptara como para-

3 Gastón Bachelard, La formación del espíritu científico (Buenos Aires: Siglo XXI, 1974).

4 Tomas Kuhn, La estructura de las revoluciones científicas (México: Fondo de cultura económica, 1962- 2011).

$5 \quad$ Kuhn, La estructura de las revoluciones científicas, 33. 
digma, "debe parecer mejor que sus competidoras; pero no necesita explicar y, en efecto, nunca lo hace, todos los hechos que se puedan confrontar con ella"6, permiten explicar la coexistencia de paradigmas. De esto se deriva que la construcción de la ciencia no es un juego de refutaciones ingenuas, sino un proceso de profundas confrontaciones, en las que, al final, no todo se develará o explicará, pero la riqueza está justamente en la lógica en que se presentan los quiebres, desde los que se pretende presentar una alternativa que contribuyera a la Enseñanza de la Teoría Cuántica.

\section{Metodología}

La metodología desarrollada para la construcción de esta reflexión toma como punto de partida las fases de la construcción de estados del arte, que se adaptaron al proceso de revisión documental realizado, para originar una fase heurística y una fase hermenéutica:

En la fase heurística, de orden preparatorio, que corresponde a la búsqueda y recopilación de fuentes de información, ${ }^{7}$ se realizó la selección del tema y la clarificación temática de los dominios del trabajo a realizar, entre libros y artículos científicos que abordan el tema de construcción del pensamiento desde una línea filosófica y aquellos que abordan la historia de las ciencias.

En la fase hermenéutica, que representa el trabajo de lectura, análisis, interpretación y comprensión crítica y objetiva, ${ }^{8}$ se concretizó una categorización inductiva, que determina tres condiciones, que constituyen el cuerpo de la reflexión: una primera condición corresponde a la contribución de documentos desde el análisis de los aportes de la Grecia Antigua: Pitágoras, Demócrito y Aristóteles; una segunda condición asume el quiebre entre el modelo geocéntrico y el modelo heliocéntrico: Ptolomeo, Galileo, Newton y, finalmente, el quiebre entre la física clásica y la teoría cuántica. Estas categorías dan cuenta de los acápites de este documento.

En la fase hermenéutica, se realizó el trabajo de análisis crítico; sin embargo, el recorrido que en esta reflexión se presenta, a través de la historia de la ciencia y la historia de la filosofía, se establece sin la pretensión de exhaustividad; lo que se procura plantear se refiere a algunos puntos de reflexión sobre quiebres epistemo-

$6 \quad$ Kuhn, La estructura de las revoluciones científicas, 44.

7 Jorge Barbosa Chacón, Juan Carlos Barbosa y Margarita Rodríguez Villabona, "Revisión y análisis documental para estado del arte: una propuesta metodológica desde el contexto de la sistematización de experiencias educativas", Investigación Bibliotecológica: archivonomía, bibliotecología e información, vol. 27. No. 61, (2013): 83-105.

8 Barbosa et al., Revisión y análisis documental para estado del arte, 91. 
lógicos que se han suscitado a lo largo de la historia y cómo estas rupturas podrían aportar a la enseñanza de la Teoría Cuántica.

\section{Planteamiento del problema}

La enseñanza de la Teoría Cuántica se articula a la enseñanza de la Estructura Atómica de la Materia. En Colombia, José Luis Villaveces ha catalogado la forma como se aborda esta temática de descriptiva, cronológica y con la construcción de prototipos planetarios de los modelos atómicos ${ }^{9}$, sin que se llevase a una reflexión sobre la construcción de las teorías en el marco de la naturaleza de la ciencia, situación que corrobora nuevamente María Alzate, como una enseñanza simplista y superficial, así como es simplista, según la autora, la forma como se aborda el tema en los libros de texto. ${ }^{10}$

Esta enseñanza simplista se deriva de no abordar la Teoría cuántica en la enseñanza de la Estructura Atómica de la Materia, lo que genera un proceso incompleto e incluso da lugar a errores conceptuales. ${ }^{11}$ Es necesario establecer esta precisión, pues, en Colombia, los Estándares Curriculares no contemplan como obligatoria esta temática. Por ello, en esta reflexión, se asume que enseñar ciencias debe alejarse de un fin meramente propedéutico, de la descripción y de la simplicidad; por el contrario, es necesario proyectar la enseñanza en el plano de la reflexión sobre la ciencia y la forma cómo se construye.

La enseñanza de la Teoría Cuántica es la disculpa investigativa que lleva a proyectar el análisis sobre la relevancia de abordar como punto de partida los quiebres epistemológicos necesarios en los cambios de paradigma que la historia de las ciencias plantea. Una enseñanza superficial y simplista tampoco aborda temas socio-científicos, por lo que resulta necesario este abordaje para el desarrollo de un pensamiento crítico $^{12}$ y sobrepasar los límites de un enfoque propedéutico.

9 José Luis Villaveces, “La enseñanza de la estructura de los átomos y las moléculas”. Tecné, Episteme y Didaxis. No. 9 (2001): 108-18.

10 María Alzate Cano, "Elemento, sustancia simple y átomo: tres conceptos problemáticos en la enseñanza y aprendizaje significativo de conceptos químicos". Revista Educación y Pedagogía, vol. 17. No. 43 (2009): 177-93.

11 Jordi Solbes, et al., "Errores conceptuales en los modelos atómicos cuánticos". Enseñanza de las Ciencias, vol. 5. No. 3 (1987): 189-95.

12 Jordi Solbes, "Contribución de las cuestiones socio-científicas al desarrollo del pensamiento crítico (I): Introducción", Eureka, vol. 10. No. 1 (2013): 1-10. 


\section{Desarrollo de la reflexión}

En esta configuración, es necesario plantear que, al tomar como marco de referencia a Kuhn y la forma como asume el proceso de construcción de la ciencia, se da un salto al tradicional modo de entender la labor científica como una dinámica entre la experimentación, los datos, las hipótesis, las leyes y las teorías. La epistemología de Tomas Kuhn remite a una nueva imagen de la ciencia, llena de disrupciones y quiebres, que se necesitan para proponer situaciones reflexivas en la enseñanza.

\subsection{Desde la antigua Grecia}

En este orden de ideas, resulta oportuno establecer un breve recorrido por algunos pensadores que construyeron las ideas centrales de la antigua filosofía griega y se acentuaron en el pensamiento científico, pues tener en cuenta esta construcción enriquece un proceso de enseñanza de la Teoría Cuántica, ya que contextualiza en el origen de las bases de la Física Clásica. Así, en la Antigua Grecia - llamada la cuna de la civilización-y, por tanto, la cuna del pensamiento occidental, se construyó, desde la filosofía, una forma de entender el mundo y, asimismo, los fenómenos naturales.

Una de esas ideas es justamente la existencia de uniformidades, que permitirían el orden del universo; esta idea desencadenó no solo el desarrollo filosófico, sino la explicación de hechos relacionados con la vida y la naturaleza en general. Desde Tales de Mileto (624-546), Anaximandro (610-545), Heráclito (535-489), de paso por Demócrito (460-370 a. C.), hasta llegar a Galileo (1564-1642) y Newton (16421727), entre otros, las ideas derivadas de la uniformidad, tales como causalidad y orden, han sido parte del pensamiento y de la forma de ver y entender la realidad.

A continuación, se presentan algunos ejemplos: en el caso de los pitagóricos, para quienes los números, concebidos como masas, se hallan constituidos por dos elementos, uno determinado o delimitado y otro indeterminado o ilimitado. En cada número, uno de estos atributos será predominante; en el caso de los pares, predomina el indeterminado, por lo que son menos perfectos que los impares, en los que predomina lo determinado; los pares son femeninos y los impares masculinos. ${ }^{13}$ En estas aserciones, se evidencia la tendencia hacia el determinismo, al orden como búsqueda de la perfección y se señala el indeterminismo como un atributo de lo imperfecto.

13 Giovani Reale y Dario Antiseri, Historia del pensamiento filosófico y científico (Barcelona: Herder, vol. 1, 1988). 
Esta posición se propone se la contemplara en un proceso de enseñanza de la Teoría Cuántica, en un acápite introductorio, pues permite comprender que, desde entonces, se plantea un adjetivo de perfección para el determinismo y de imperfección para lo indeterminado que, de una u otra forma, se ve proyectado hoy en día en el predominio de un pensamiento determinista. Por otra parte, la tradición filosófica señala a los pitagóricos como los primeros en denominar Cosmos al universo, que dedujeron de la idea de número y su correspondencia con el orden, de donde se infiere un universo ordenado.

Ahora bien, en la enseñanza de la Estructura de la Materia, se alude a Demócrito, también de la Antigua Grecia, como el precursor del atomismo, pero es necesario comprender que Demócrito introduce los átomos como pequeñas masas de materia, las que se encuentran en una cantidad infinita en el universo, que pueden "ser analizables en partes desde el punto de vista lógico (cosa desprovista de interés, según él), pero físicamente indivisibles". ${ }^{14}$ De esta forma, se comienza a construir la idea de dos tipos de movimiento atómico. Demócrito, plantea el movimiento rectilíneo, por el cual los átomos colisionan, y el movimiento circular, como un vórtice cósmico, que lleva a las transformaciones del Universo, determinables entre los átomos, sin necesariamente obedecer a un plan. ${ }^{15}$

Si bien estas ideas atomistas corresponden a un mundo al azar, está claro que le asignan una causa a su surgimiento. Según los atomistas, el orden "es el resultado de un encuentro mecánico entre los átomos y no algo proyectado o producido por una inteligencia"16. Se presenta como una nueva forma de entender el universo; sin embargo, la causalidad y el determinismo también siguen siendo un principio de la filosofía atomista.

El atomismo de Demócrito se constituye en una disrupción en la concepción griega del universo, por cuanto plantea que los átomos llevan consigo, de forma inherente, el movimiento y la interacción entre ellos origina los cuerpos infinitos que existen en el universo; además, en el universo atomista, todo sucede como un producto de una concatenación causal, que envuelve lo existente: "la interpretación del mundo desde una mecánica atomista entra en una tensión interna con la concepción específicamente griega del orden de la naturaleza"; ${ }^{17}$ del mismo modo, según los aristotélicos, esta teoría no podía justificar la varie-

14 Hilary Armstrong, Introducción a la filosofía antigua (Buenos Aires: Eudeba, 1996), http:// www.sagrado corazon.edu.ar/web/sexto_elect_a/Filosofia/IntroFilosofiaAntigua.pdf

15 José Luis Calabrese, "Ampliando las fronteras del reduccionismo. Deducción y sistemas no lineales", Psicoanálisis AP de BA, vol. 21. No. 3 (1999): 431-53.

16 Reale y Antiseri, Historia del pensamiento filosófico y científico, 70.

17 Hans Georg Gadamer, El inicio de la sabiduría (Barcelona: Paidós, 2001), 9. 
dad de sustancias existentes y no se podía justificar el movimiento, al plantear la idea de vacío.

En la enseñanza de la Estructura Atómica de la Materia, se debería resaltar que: 1) la idea de Demócrito sobre el átomo como una entidad filosófica y la idea de átomo para el atomismo moderno son muy diferentes, 2) Dalton no establece una retoma de conceptos de Demócrito, por tanto no se puede ignorar el sistema conceptual en el que se construyen estos conceptos, ${ }^{18}$ 3) Demócrito buscaba fundamentar al atomismo como un sistema filosófico, mientras tanto, en el atomismo moderno de Dalton, los átomos surgen físicamente desde la ley de las proporciones múltiples; por tanto, las leyes de proporcionalidad de Dalton y Proust no son ajenas a la Teoría Atómica.

Una vez planteadas las ideas de Demócrito, es necesario abordar a Aristóteles (384-322 a. C.), quien mostró un interés por las ciencias empíricas y la recolección de datos, también de corte empírico; incluso plantea una clasificación de ciencia en tres ramas: teóricas, prácticas y productivas, siendo las más importantes las primeras, por buscar el saber por sí mismo, ${ }^{19}$ lo que, sin duda, tendrá un impacto importante en las posiciones epistemológicas de entonces y de este momento con respecto a cómo se concibe la ciencia y el estatuto de cientificidad.

Aristóteles inició los estudios sistemáticos de física, biología y ciencias naturales en general, que derivan de principios filosóficos y lógicos; entre los filosóficos, se encuentran el empirismo, el hilemorfismo, el intelectualismo, el teleologismo, el animal político que tiene logos, energeia; los principios lógicos son la no contradicción, la identidad, el tercio excluido y la razón suficiente. ${ }^{20}$

Es importante analizar con detenimiento la idea de Aristóteles en torno a lo que es el principio como causa del movimiento y de su perfección o realización, así como, también, de su generación, ordenamiento y cognoscibilidad. La idea de causa es "lo primero", donde subyace el concepto de principio, que se establece tanto para los seres como para su conocimiento. ${ }^{21}$ Este planteamiento causal ha permeado hasta los días actuales; nótese la causa como concepto de principio, ligado a un orden que, además, se especifica en la cognoscibilidad. Esto permite relacionar estos planteamientos con el pensamiento occidental moderno y se puede inferir

18 Alzate Cano, "Elemento, sustancia simple y átomo", 177-93.

19 Reale y Antiseri, Historia del pensamiento filosófico y científico, 164.

20 Sebastián Salgado González, "La filosofía de Aristóteles", Cuadernos Duereiras, Red Universitaria de Aprendizajes. (2012), http://repositoriodigital.academica.mx/jspui/handle/987654321/483831

21 Salgado González, La filosofía de Aristóteles. 
que tiene su cuna en la cosmovisión aristotélica y, a su vez, ha sido el marco de la racionalidad con que se constituyó la ciencia clásica y cómo se enseña.

Aunque la cosmovisión aristotélica no era la única idea respecto a cómo se organizaba el universo, puesto que, como se enunció antes, los pitagóricos, en primera instancia, plantearían la idea de que el universo no girara en torno de la Tierra, sino alrededor de una bola de fuego y, desde el atomismo de Demócrito, la idea de átomos en movimiento y separados por regiones vacías, implicaba que la "Tierra era uno más entre los cuerpos celestes, que no estaba en reposo y no era el centro del universo"; ${ }^{22}$ estos planteamientos no tuvieron el impacto necesario y las concepciones aristotélicas prevalecieron hasta la Edad Media, incluso con la idea del geocentrismo.

Sin embargo, acudir a una interpretación de estas formas de pensamiento y su incidencia en el pensamiento actual requiere alejarse de ellas como sentencias declarativas y concentrarse en las "condiciones en que se ha transmitido el texto en cada caso, pues esas condiciones nos permiten el acceso a los textos que leemos como fragmentos". ${ }^{23}$ Esta es una descripción de lo que se denominaría filosofía de la physis o filosofía de los físicos, que caracteriza una mentalidad científica clásica.

Este recorrido por la filosofía de la Grecia antigua es un preámbulo para situarse en el origen del pensamiento y es posible abordarlo en la clase de ciencias, y específicamente de enseñanza de la Teoría Cuántica, ya que genera un escenario importante para combatir las ideas ingenuas y motivar hacia un pensamiento crítico y reflexivo en los estudiantes con respecto a cómo se construyen los conceptos y la naturaleza de la ciencia.

\subsection{Del geocentrismo al modelo heliocéntrico: una gran revolución científica y ontológica}

Se han esbozado algunos puntos de confluencia entre el mundo filosófico y la comprensión de los fenómenos naturales y, en esta dinámica, dada la imposibilidad de conjugar siglos y siglos de construcción del conocimiento, es necesario concentrarse en una revolución científica fundamental en la historia de la humanidad: el periodo en que la concepción "geocéntrica", basada en las nociones anteriormente descritas, comenzara a tambalear. Esta revolución, que configura un gran quiebre epistemológico, se aborda tangencialmente en la enseñanza de las ciencias, sin en-

22 Germán Guerrero Pino, "El paso del geocentrismo al heliocentrismo", El hombre y la máquina. No. 22 (2004), 104.

23 Gadamer, El inicio de la sabiduría, 35. 
fatizar que trajo consigo cambios en los métodos y, más que en los métodos, en la posibilidad de ver cosas distintas con los mismos instrumentos y en los mismos lugares; se trata de observar los mismos objetos bajo una luz diferente; ${ }^{24}$ esta inferencia ayudaría a los estudiantes a comprender la necesidad de algunas rupturas, tanto en el conocimiento como en la interpretación de los fenómenos naturales.

Para comprender mejor este punto, es necesario retomar a Aristóteles, quien basó la teoría geocéntrica en dos principios: "el principio de circularidad y uniformidad, y el principio de diferencia entre mundo terrestre y celeste". ${ }^{25}$ Estos principios encierran una concepción ontológica y antropológica que deriva prácticamente en una teología: la tierra como centro del universo.

La mirada geocéntrica originó una serie de modelos explicativos sobre el origen del hombre y su lugar en el universo; Ptolomeo (100-160), por ejemplo, logró, en su época, asombrosos cálculos, que ajustó a dicha mirada; explicó el movimiento retrógrado de los planetas. Los cálculos que extraordinariamente logró resultaban contradictorios respecto al modelo geocéntrico, pero sus convicciones le impidieron ver los alcances de sus investigaciones y se produjo una acomodación de esos datos al modelo geocéntri$\mathrm{co}^{26} \mathrm{El}$ trabajo realizado por Ptolomeo y los "ajustes" que introdujo a sus datos muestran que la ciencia y el conocimiento científico son parte de un contexto socioeconómico, político y religioso y que el mundo natural es el resultado de quien lo interpreta.

En muchas ocasiones, los estudiantes pueden preguntarse: ¿por qué un modelo, que ahora se ve como ingenuo y supersticioso, perduró durante siglos? Entonces, se necesitaría explicar que la cosmología aristotélica había sobrevivido sin mayores problemas durante mucho tiempo. Tanto astrónomos como navegantes, durante casi quince siglos, se sirvieron de este modelo que, además, se ajustaba a las concepciones teológicas y sociales del momento, para constituirse, en términos de Kuhn, en un paradigma con un gran poder explicativo.

Por tanto, en la enseñanza no podría presentarse este periodo como una transformación casi ingenua de un modelo a otro, pues se genera una visión simplista de la naturaleza de las ciencias; por el contrario, se requiere enfatizar en que se trata de una crisis, entre otras cosas, de las concepciones antropológicas, teológicas y sociales, y que se ajusta a lo que Kuhn plantea después de una revolución científica: responder a un mundo diferente. ${ }^{27}$

24 Kuhn, La estructura de las revoluciones científicas, 44.

25 Guerrero Pino, "El paso del geocentrismo al heliocentrismo", 100.

26 Guerrero Pino, "El paso del Geocentrismo al Heliocentrismo", 98-110.

27 Kuhn, La estructura de las revoluciones científicas, 44. 
Es posible, además, explicar que "solo el filtro de los años y no la regulación de los hombres puede decidir el valor de las distintas contribuciones", ${ }^{28}$ pues se necesitaron muchos años de trabajo alrededor del nuevo paradigma para que el modelo heliocéntrico pudiera establecerse de alguna forma; esto, en el plano de la enseñanza, posibilita debates y reafirma que los temas sociocientíficos contribuyen a la formación de un pensamiento crítico. ${ }^{29}$

En este contexto, otro aspecto que contribuye a una enseñanza apegada a los quiebres epistemológicos se configura en siglo XVII, con el surgimiento de un nuevo espíritu científico, con Francis Bacon, Galileo Galilei y René Descartes, en el que se condensaron la relevancia de la experiencia y la teoría, el status del formalismo matemático y la autonomía de la ciencia con respecto a la filosofía y la fe. Aquí, también, el determinismo científico y filosófico comenzó a imponerse; ${ }^{30}$ estos científicos y filósofos asumieron que la explicación de los fenómenos naturales se haría de manera "objetiva".

Este es el comienzo de otra gran revolución. La noción de causa, según Galileo, que transforma la idea de causalidad de la Grecia Antigua, es un paso de una causalidad ontológica, eficiente y metafísica, a una causalidad lógico-fenoménica, formal-nomológica y físico-factual. ${ }^{31}$ Esta crisis en la noción de causalidad tendrá repercusiones profundas, no solo en el conocimiento, sino en la forma cómo se asumirían ahora los fenómenos naturales y los elementos tanto físicos como conceptuales utilizados para su interpretación.

Galileo buscó la causa de los fenómenos naturales e instauró, además, la transformación en la naturaleza de la Física como ciencia, tanto en su método como en su contenido. Hasta entonces, la Física era cualitativa y esencialista, sin la idea de precisión, pero, ahora, sería una ciencia cuantitativa, en busca de resultados precisos, que permitieran alcanzar el conocimiento de la naturaleza ligado a una visión matemática. ${ }^{32}$ Esto permite inferir no solo la noción de causalidad, en Galileo, sino las derivaciones epistemológicas que le subyacen a una teoría y la complejidad que encierra y sobrepasa los límites de disciplina científica.

28 Gerald James Holton y Stephen Brush, Introducción a los conceptos y teorías de las ciencias físicas (Barcelona: Reverté, 1997), 291.

29 Jordi Solbes, et al., "Debates y argumentación en las clases de Física y Química”, Alambique. No. 63 (2010): 65-76.

30 Ignacio A. Silva, Indeterminismo en la naturaleza y mecánica cuántica. Tomás de Aquino y Werner Heisenberg (Pamplona: Universidad de Navarra, 2011), https://dadun.unav.edu/bitstream/10171/36909/1/ 201602\%20CAF\%20232\%20\%282011\%29.pdf

31 Rafael Martínez, "La filosofía de Galileo y la conceptualización de la causalidad física", Thémata. No. 14, (1995): 37-59.

32 Martínez, "La filosofía de Galileo y la conceptualización de la causalidad física”, 37-59. 
Ahora bien, Isaac Newton asumió la posibilidad de leyes universales, que permitieran explicar el orden de los sistemas físicos o sociales. Así, las ecuaciones de dinámica lineal son la base de la visión mecanicista, según la cual existen leyes generales e inmutables y, también, la base de una concepción determinista, en la que "la precedencia de un estado condena al mundo a su estado subsiguiente". ${ }^{33}$ Este acápite, trasladado a la enseñanza, permite introducirse en el paradigma que constituye la mecánica clásica, bajo la cual se buscan ecuaciones para la explicación de la realidad y, en esta, las leyes físicas son universales, con lo que se consolida un periodo de ciencia normal.

Sin embargo, la calma comenzaría nuevamente a tambalear cuando resultó que algunos fenómenos de la naturaleza no podían explicarse a partir de la Física Clásica. El comportamiento corpuscular de la luz y fenómenos asociados a ondas, como la radiación del cuerpo negro, el efecto fotoeléctrico y el mundo atómico, para mencionar solo algunos, no tenían una explicación satisfactoria y generarían grandes interrogantes para los científicos de finales del siglo XIX e inicios del siglo XX. Se revelaban limitantes de la Física Clásica y esta nueva revolución requirió de un "replanteamiento global con un nuevo marco conceptual" ${ }^{34}$ y, de nuevo, no se trata solo del campo científico, sino de la comprensión de la naturaleza, de sus fenómenos y de la realidad, con una nueva forma de pensamiento, que marcaría un verdadero quiebre epistemológico, sin precedentes.

Este recorrido histórico, que vincula a la ciencia y la filosofía, permitiría a los docentes constituir en el aula un diálogo de saberes que, a su vez, desmitifica la idea de ciencia y permite construir una imagen más plural y contextualizada. ${ }^{35}$

\subsection{Una revolución que replantea la forma de pensar}

Asumir los aportes de la Grecia Antigua y el quiebre del modelo heliocéntrico ante el modelo geocéntrico, en la enseñanza, permiten comprender que, hasta entonces, no afectaban la base del pensamiento occidental, descrita en la parte inicial de esta reflexión. Muchos de los planteamientos aristotélicos permanecen en la Física Clásica: causalidad y determinismo, pero, desde hace más de un siglo, esa "zona de confort", en la que se encontraba la ciencia, vuelve a quedar en la

33 Roberto Ávila Ayala, "Aproximación al concepto de determinismo". Cuestiones de Filosofía. No. 10 (2012), 124, https://doi.org/10.19053/01235095.646

34 Jordi Solbes y Vicente Sinarcas, “Una propuesta para la enseñanza aprendizaje de la física cuántica basada en la investigación en didáctica de las ciencias", Revista de enseñanza de la física, vol. 23. Nos. 1 y 2 (2011): 57-84.

35 Zulman Muñoz y Sandra Cerón, "Formación de un espíritu científico en la Educación Básica desde la enseñanza de las ciencias naturales", Tendencias, vol. 16. No. 1 (2015), 147-58. 
cuerda floja. Una vez más, la razón se confunde ante nuevas evidencias y una ciencia revolucionaria se instaura; se trata de una ciencia que contrarresta principios fundamentales del entendimiento humano, el orden, la causa, la certeza y el determinismo, especialmente.

Abordar, en la enseñanza, el determinismo, desde su origen, permite comprender que es un problema científico y ha sido una postura social incluso ante el mismo conocimiento, al asumir que "la probabilidad es una medida de nuestra falta de conocimiento" ${ }^{\prime 36} \mathrm{y}$, por tanto, era el mayor avance del conocimiento para el pensamiento clásico y para la ciencia que se ha heredado. La mecánica clásica constituye un gran paradigma, que permite la explicación de muchos de los modelos científicos, no solo todavía vigentes, sino ofreció la posibilidad de predecir desde el movimiento de una partícula hasta el destino final de galaxias y estrellas; sin embargo, fenómenos que tenían que ver con la luz, emisión de radiación, la misma estructura y naturaleza de la materia, harían tambalear el acervo explicativo de esa mecánica clásica.

Este problema se pone en el foco de la discusión; ahora, la realidad es más ininteligible, enmarañada de fenómenos que se resisten a las explicaciones clásicas, lo que, sin duda, no significa que los métodos mecanicistas y reduccionistas hubieran fracasado o resultasen incorrectos, pero es claro que no todos los fenómenos de la naturaleza responden al orden y regularidad establecidos. ${ }^{37} i$ Sería acaso que la estructura de la materia y la naturaleza de las radiaciones, la distribución de las líneas espectrales, la radiación del cuerpo negro y muchos otros resultados experimentales requieren de un nuevo conjunto de principios que permitieran formalizar el comportamiento no clásico de un número creciente de procesos físicos?

Sí, pues estos nuevos fenómenos escapan a la anhelada predicción; introducen, a la vez, la noción de la probabilidad en la naturaleza, algo que no tenía cabida en el mundo físico, desde los filósofos griegos hasta los inicios del siglo XX. A esta probabilidad no la producía la indeterminación de las leyes naturales, sino la naturaleza, en los casos en los que "hay una alta sensibilidad a las condiciones iniciales". ${ }^{38}$ Por tanto, en el aula de clases, al abordar el contexto descrito en esta reflexión, es posible plantear un interrogante: i... esto significaría la caída del determinismo y el surgimiento del indeterminismo? No necesariamente. El determinismo clásico se aplicaría al mundo clásico y el indeterminismo a un nuevo mundo, el mundo

36 Calabrese, "Ampliando las fronteras del reduccionismo. Deducción y sistemas no lineales", 437.

37 Ávila Ayala, "Aproximación al Concepto de Determinismo", 124, https://doi. org/10.19053/01235095. 646

38 Ávila Ayala, “Aproximación al Concepto de Determinismo", 130. 
cuántico, con objetos nuevos, los objetos cuánticos, y una nueva teoría, la Teoría Cuántica. Asumir este planteamiento para los estudiantes es fundamental; permite comprender que tanto la física clásica como la cuántica están vigentes, pero explican fenómenos diferentes.

Al respecto, el mismo de Broglie, al referirse a la Teoría Cuántica, señaló: "Su aparición en la Ciencia ha producido en ella una revolución conceptual cuya importancia no medimos aún con exactitud, pero que, sobrepasando con mucho la realizada por la teoría de la Relatividad, constituye uno de los cambios más importantes en la historia del pensamiento humano". ${ }^{39}$ Esto implicaría, sin duda, nuevas lógicas, nuevas formas de ver la realidad, si es que se puede hablar de realidad o, mejor, debería decirse nuevas realidades. Comienza a surgir una nueva ciencia, que, al ser muy exitosa en los resultados experimentales, a la vez resulta paradójicamente incapaz de interpretar esos resultados y las predicciones, ${ }^{40}$ lo que, según muchos, la establece como una teoría incompleta.

Una nueva ciencia en la que, además de los problemas filosóficos referidos al determinismo y la causalidad, relacionados, asimismo, con el método de producción científica, debería enfrentar la adopción de soluciones de tipo estadístico y probabilístico. Sin embargo, este no era un asunto nuevo, pues ya Bernoulli, desde la hidrodinámica, y Gauss, en la teoría cinética de los gases, adoptaban métodos estadísticos y probabilísticos, sin que esto significara la presunción del indeterminismo sencillamente constituía unas herramientas para la interpretación. ${ }^{41}$

El indeterminismo se relaciona con conceptos tales como la dualidad onda-partícula, la incertidumbre, el problema de la medida y el pensamiento complementario. Mientras la mecánica clásica trasladaba a un sistema en que el observador no forma parte del fenómeno, la mecánica cuántica asume no solamente que el observador constituye una parte del instrumento de medida y afecta al fenómeno estudiado, sino corresponden a un mismo sistema; por tanto, no es una relación simple de parte y todo o suma de las partes, no se puede hablar de unidad o de multiplicidad. La medida, en esta teoría, es un sistema rizomático, que se traduce en un problema de superposición: "haciendo que la indeterminación que caracteriza al mundo cuántico se convierta también en parte del mundo al nivel clásico"; ${ }^{42}$ así,

39 Silva, Indeterminismo en la naturaleza y mecánica cuántica, https://dadun.unav.edu/bitstream/10171/36909/1/201602\%20CAF\%20232\%20\%282011\%29.pdf

40 Ana Vallejo Clavijo, "Problemas epistemológicos en torno a la física cuántica", Hallazgos, vol. 2. No. 4 (2015): 96-103.

41 Silva, Indeterminismo en la naturaleza y mecánica cuántica.

42 Favio Cala Vitery y Edgar Castañeda. Mecánica cuántica. Sobre su interpretación, historia y filosofía (Bogotá: Universidad Jorge Tadeo Lozano, 2011). 
en la Teoría Cuántica, cualquier interacción de un ente cuántico con uno clásico se considera observación. ${ }^{43}$

Por otra parte, en las ciencias aplicadas, como la astronomía, la meteorología, la biología poblacional y las ciencias económicas, el caos constituye un objeto de estudio, ${ }^{44}$ lo que deja en muy mala posición el determinismo de Laplace, que aseguraba la posibilidad de predicción de todo el universo. Hoy, esta pretensión no resiste al principio de indeterminación, de Heisenberg, que, además, se sustenta en la fascinación de las matemáticas. Lo que se observa no son propiedades de la naturaleza, sino el resultado de la interrelación del observador con esa naturaleza. Por otra parte, Niels Bohr insistió en que: "no es posible saber cómo es la naturaleza, sino cómo se manifiesta, cuando se forma parte del experimento".

Asumir, en la enseñanza, los quiebres epistemológicos descritos, permite allanar el terreno para comprender que la Teoría Cuántica no se trata de una generalización. El indeterminismo se eleva a principio, solo para el mundo cuántico; permite entender que, en la dinámica de construcción de la ciencia, el cambio de los paradigmas incluye una verdadera revolución, que trae profundas transformaciones epistemológicas, culturales, sociales y antropológicas, además de la creación de nuevos conceptos, que sostuvieran el nuevo paradigma, con la apertura a una reflexión respecto a cómo llevar esta "revolución" al aula de clases.

\section{CONCLUSIÓN}

Esta forma de pensar se constituyó durante siglos de forma paralela al desarrollo sociocultural, político, religioso y científico, lo que lleva a que las revoluciones científicas afectasen la explicación que se da sobre la realidad. Si bien existen diferencias sustanciales entre la idea de Aristóteles, de los pitagóricos y de Demócrito respecto a los fenómenos naturales, la idea de causalidad, determinismo y orden prevalecen en estas posturas. Por ello, el atomismo de Demócrito no se configuró como un verdadero quiebre; además, no logró instaurarse como un paradigma en su época y su idea de vacío y movimiento no se avalaron.

El cambio del modelo geocéntrico al modelo heliocéntrico se constituye en un quiebre epistemológico y, aunque generara una transformación en la concepción del cosmos y el lugar del ser humano en él, no hay una disrupción en el pensamiento causal y determinista. Comprender que desde la Grecia Antigua se dio al deter-

43 Levi Landau y Yevgueni Liftshitz, Curso abreviado de Física Teórica: Mecánica Cuántica (4ํㅡㄹ ed. Moscú: Mir, 1974).

44 Leonard Smith, Caos: una breve introducción (Madrid: Alianza Editorial, 2011). 
minismo un adjetivo de perfección permite asumir por qué es tan difícil aceptar el indeterminismo.

Tanto el atomismo de Demócrito, como el advenimiento del modelo heliocéntrico, y el determinismo y objetividad de la física clásica, son escenarios que, llevados al aula de clase, permitirían allanar el terreno para la enseñanza de la Teoría Cuántica; conocer que han existido quiebres importantes en la ciencia y en la filosofía, así como la naturaleza que comporta el trabajo de los científicos y cómo afecta y se ve afectado por el entorno sociocultural, económico, político y social, permite sobrepasar la barrera de una enseñanza simplista o demasiado formalista.

Es necesario enfatizar en que la revolución producida por el desarrollo de la Teoría Cuántica no solo implicaría una explicación diferente de los fenómenos naturales, sino un nuevo pensamiento, regido por el indeterminismo y la probabilidad, así como la existencia de objetos distintos a los que se estaba acostumbrados: los objetos cuánticos, pero ¿cómo se hablaría de una nueva ciencia, si no se reconoce el proceso de la ciencia clásica?, ¿cómo se habla de una nueva ciencia para un nuevo mundo, el mundo cuántico, si no se comprende el origen del mundo clásico?, ¿cómo se reconoce que se requiere un quiebre, si no se da cuenta de los quiebres que se han requerido en la historia?

Es imperioso, en la enseñanza, asumir que la verticalidad, la linealidad y el determinismo, entre otros, son conceptos que, en su tiempo y contexto, impulsaron formas de conocimiento, pero las realidades actuales exigen otras racionalidades para desenvolverse. La posibilidad de enseñar desde la dinámica de la ciencia, que propuso Kuhn: rupturas y revoluciones que planteasen la necesidad de un quiebre epistemológico, aporta a la enseñanza de la Teoría Cuántica un espacio de discusión sobre su origen y el origen del pensamiento que ha limitado su comprensión. El debate, la reflexión y el análisis en torno a la historia de las ciencias y la filosofía generan un espacio de cimentación, en que los estudiantes asumen la complejidad de la construcción de las teorías y la posibilidad de desarrollar un pensamiento crítico. 


\section{REFERENCIAS}

Alzate Cano, María. "Elemento, sustancia simple y átomo: tres conceptos problemáticos en la enseñanza y aprendizaje significativo de conceptos químicos", Revista Educación y Pedagogía, vol. 17. No. 43 (2009): 177-93.

Armstrong, Hilary. Introducción a la filosofía antigua. Buenos Aires: Eudeba, 1996. http://www.sagrado corazon.edu.ar/web/sexto_elect_a/Filosofia/IntroFilosofiaAntigua.pdf

Ávila Ayala, Roberto. "Aproximación al concepto de determinismo", Cuestiones de Filosofía. No. 10 (2012), 120-34. https://doi.org/10.19053/01235095.646

Bachelard, Gaston. La formación del espíritu científico. Buenos Aires: Siglo XXI, 1974.

Barbosa Chacón, Jorge, Juan Carlos Barbosa y Margarita Rodríguez Villabona. "Revisión y análisis documental para estado del arte: una propuesta metodológica desde el contexto de la sistematización de experiencias educativas", Investigación Bibliotecológica: archivonomía, bibliotecología e información, vol. 27. No. 61 (2013): 83-105.

Cala Vitery, Favio y Edgar Castañeda. Mecánica cuántica. Sobre su interpretación, historia y filosofía. Bogotá: Universidad Jorge Tadeo Lozano, 2011.

Calabrese, José Luis. "Ampliando las fronteras del reduccionismo. Deducción y sistemas no lineales", Psicoanálisis AP de BA, vol. 21. No. 3 (1999): 431-53.

Gadamer, Hans Georg. El inicio de la sabiduría. Barcelona: Paidós, 2001.

Guerrero Pino, Germán. "El paso del geocentrismo al heliocentrismo", El hombre y la máquina. №. 22 (2004): 98-110.

Holton, Gerald James y Stephen Brush, Introducción a los conceptos y teorías de las ciencias físicas. Barcelona: Reverté, 1997.

Kuhn, Tomas. La estructura de las revoluciones científicas. México: Fondo de cultura económica, 19622011.

Landau, Levi y Yevgueni Liftshitz. Curso abreviado de Física Teórica: Mecánica Cuántica. Moscú: Mir, 1974.

Martínez, Rafael. "La filosofía de Galileo y la conceptualización de la causalidad física”, Thémata. No. 14, (1995): 37-59.

Muñoz, Zulman y Sandra Cerón. "Formación de un espíritu científico en la Educación Básica desde la enseñanza de las ciencias naturales", Tendencias, vol. 16. No. 1 (2015), 147-58.

Reale, Giovani y Dario Antiseri. Historia del pensamiento filosófico y científico. Barcelona: Herder, vol. $1,1988$.

Salgado González, Sebastián. "La filosofía de Aristóteles", Cuadernos Duereiras, Red Universitaria de Aprendizajes. (2012). http://repositoriodigital.academica.mx/jspui/handle/987654321/483831

Silva, Ignacio A. Indeterminismo en la naturaleza y mecánica cuántica. Tomás de Aquino y Werner Heisenberg. Pamplona: Universidad de Navarra, 2011. https://dadun.unav.edu/bitstream/10171/36909/1/201602\% 20CAF\%20232\%20\%282011\%29.pdf

Smith, Leonard. Caos: una breve introducción. Madrid: Alianza Editorial, 2011.

Solbes, Jordi. "Contribución de las cuestiones socio-científicas al desarrollo del pensamiento crítico (I): Introducción”, Eureka, vol. 10, No. 1 (2013): 1-10.

Solbes, Jordi y Vicente Sinarcas. "Una propuesta para la enseñanza aprendizaje de la física cuántica basada en la investigación en didáctica de las ciencias", Revista de enseñanza de la física, vol. 23. Nos. 1 y 2 (2011): 57-84.

Solbes, Jordi et al. "Errores conceptuales en los modelos atómicos cuánticos", Enseñanza de las Ciencias, vol. 5. No. 3 (1987): 189-95.

Solbes, Jordi et al. "Debates y argumentación en las clases de Física y Química”, Alambique. No. 63 (2010): 65-76. 
Vallejo Clavijo, Ana. "Problemas epistemológicos en torno a la física cuántica", Hallazgos, vol. 2. No. 4 (2015): 96-103.

Villaveces, José Luis. “La enseñanza de la estructura de los átomos y las moléculas”, Tecné, Episteme y Didaxis. No. 9 (2001): 108-18. 